\title{
A retrospective analysis of the risk factors affecting recurrence time in patients with recurrent glioblastoma
}

\author{
Renhua Huang ${ }^{1,2}$, Tianwei Wang ${ }^{3}$, Zhijun Liao ${ }^{4}$, Zhenwei Wang ${ }^{2}$, Ming $\mathrm{Ye}^{2}$, Di Zhou ${ }^{2}$, Huaying Xie ${ }^{2}$, \\ Yongrui Bai ${ }^{2}$, Yongming Qiu ${ }^{3}$, Yulong Liu ${ }^{1,5}$ \\ ${ }^{1}$ Department of Nuclear Accident Medical Emergency, the Second Affiliated Hospital of Soochow University, Suzhou, China; ${ }^{2}$ Department of \\ Radiation Oncology, Renji Hospital, Shanghai Jiao Tong University School of Medicine, Shanghai, China; ${ }^{3}$ Department of Neurosurgery, Renji \\ Hospital, Shanghai Jiao Tong University School of Medicine, Shanghai, China; ${ }^{4}$ Department of Radiation Oncology, Shanghai International Medical \\ Center, Shanghai, China; 'State Key Laboratory of Radiation Medicine and Protection, School of Radiation Medicine and Protection, Soochow \\ University, Suzhou, China \\ Contributions: (I) Conception and design: R Huang, Y Liu; (II) Administrative support: M Ye, Y Bai, Y Qiu; (III) Provision of study materials or \\ patients: R Huang, Z Liao, Z Wei, H Xie, D Zhou; (IV) Collection and assembly of data: All authors; (V) Data analysis and interpretation: T Wang; (VI) \\ Manuscript writing: All authors; (VII) Final approval of manuscript: All authors. \\ Correspondence to: Yulong Liu. Department of Nuclear Accident Medical Emergency, the Second Affiliated Hospital of Soochow University, San \\ Xiang Road NO.1055, Suzhou 215004, China. Email: yulongliu2002@suda.edu.cn.
}

Background: This study explored the related factors that influence the recurrence time of glioblastomas (GBM).

Methods: A retrospective study of recurrent GBM patients with surgical resection was performed. Recurrence time was analyzed using Kaplan-Meier survival curves. The Cox regression model was used to investigate the possible factors associated with recurrence time.

Results: A total of 176 patients (113 males and 63 females) were enrolled in the study, with a median age of 57 years (range, 19-76 years). From this cohort, 18 patients (10.2\%) had gross total resection (GTR), 53 patients (30.1\%) had subtotal resection (STR), and 105 patients $(59.7 \%)$ had partial resection (PR). Postoperatively, all patients received radiotherapy (RT), with $55.1 \%$ administered concurrent chemotherapy (CTh) and $59.7 \%$ administered adjuvant $\mathrm{CTh}$. The median recurrence time was 10.0 months (range, 1.0-75.0 months). Patients with PR $(\mathrm{P}=0.004)$, gliomas that contacted the subventricular zone (SVZ) $(\mathrm{P}=0.004)$, isocitrate dehydrogenase 1 (IDH1) wild-type $(\mathrm{P}=0.048)$, telomerase reverse transcriptase (TERT) C228T wild-type $(\mathrm{P}=0.012)$, and positive glial fibrillary acidic protein (GFAP) expression $(\mathrm{P}=0.044)$ had a shortened time to recurrence. Cox regression analysis revealed that $\mathrm{PR}(\mathrm{P}=0.036)$, SVZ contact $(\mathrm{P}=0.008)$, and TERT C228T wild type $(\mathrm{P}=0.023)$ were significantly associated with a shortened recurrence time.

Conclusions: PR, tumor contacting the SVZ, and TERT C228T wild type were independent risk factors for tumor recurrence in patients with GBM.

Koywords: Recurrent glioblastoma; recurrence; subventricular zone (SVZ); isocitrate dehydrogenase 1 (IDH1); surgical resection.

Submitted Mar 04, 2021. Accepted for publication Apr 28, 2021.

doi: 10.21037/apm-21-823

View this article at: http://dx.doi.org/10.21037/apm-21-823

\section{Introduction}

Glioblastoma (GBM) is the most frequent malignancy and the most aggressive type of primary brain tumor (1). It accounts for approximately $48 \%$ of all primary malignant central nervous system tumors $(2,3)$. At present, comprehensive therapies for the management of GBM include surgery, chemoradiotherapy, and immunotherapy (4). Although there are many treatment options for GBM 
patients, the outcome is dismal, and GBM typically recurs during the first year $(5,6)$. In this study, a correlation analysis was conducted to determine the prognostic risk factors affecting the time to recurrence in GBM patients. We present the following article in accordance with the STROBE reporting checklist (available at http://dx.doi. org/10.21037/apm-21-823).

\section{Methods}

\section{Patient selection}

A retrospective study was conducted on patients who were histologically diagnosed with GBM and underwent surgery at the Department of Radiotherapy of Renji Hospital, Shanghai Jiao Tong University School of Medicine between June 2008 and August 2020. Data for 426 patients were extracted from the database archives. The following inclusion criteria were applied: adult patients with newly diagnosed intracranial lesion who underwent surgical resection of the tumor; the pathological diagnosis of GBM was confirmed by at least two pathologists; and all basic data (including clinical characteristics, medical records, preoperative and tumor recurrence neuroimaging, molecular markers) and follow-up data were readily available. The following patients were excluded: patients less than 18 years old; patients who underwent biopsy; patients with other organic tumors; and patients in whom the tumor recurrence time was unavailable. Ultimately, 176 patients were enrolled in this study. All procedures performed in this study involving human participants were in accordance with the Declaration of Helsinki (as revised in 2013). This study was approved by the Ethics Committee of Renji Hospital, Shanghai Jiaotong University School of Medicine (No. RA-2020-056). Individual consent for this retrospective analysis was waived.

\section{Data extraction}

Patient demographic data (including gender and age), clinical characteristics [including Karnofsky performance status (KPS), tumor diameter, tumor volume, location, tumor contact with the subventricular zone (SVZ), and enhanced features], extent of resection (EOR), management after surgery [such as radiotherapy (RT), chemotherapy (CTh), or concurrent RT and CTh], and time to recurrence were collated. For data analysis, patients were divided into the following 2 groups according to the median age: $\leq 57$ years and $>57$ years. The KPS was categorized into 2 groups according to the median value, namely, $<90$ and $\geq 90$. The proliferation of Ki-67 was classified into the following 2 groups: Ki-67 $<0.3$ and Ki- $67 \geq 0.3$. The tumor diameter and volume were classified into 2 groups, namely, $\leq 4.7 \mathrm{~cm}$ and $>4.7 \mathrm{~cm}$ for diameter and $\leq 33$ and $>33 \mathrm{~cm}^{3}$ for volume. The tumor sites were obtained from preoperative magnetic resonance imaging (MRI), and EOR was evaluated through postoperative MRI or computed tomography by experienced neurosurgeons and radiologists. Gross total resection (GTR) was defined as $>90 \%$ removal, subtotal resection (STR) was defined as $80-90 \%$ removal, and partial resection (PR) was defined as $<80 \%$ removal. The EOR was classified into 2 groups, namely GTR/STR and PR. Postoperative adjuvant therapies included RT and CTh. Pathologic immunohistochemistry data were confirmed by two senior pathologists, with particular focus on the expression of the following biomarkers: isocitrate dehydrogenase (IDH) 1 , telomerase reverse transcriptase (TERT) T228C, Ki-67, glial fibrillary acidic protein (GFAP), O6-methylguanineDNA methyltransferase (MGMT), tumor protein P53 (TP53), ATRX (alpha-thalassemia/mental retardation, $\mathrm{X}$-linked gene), and the B-Raf gene mutation BRAF V600E. The time to recurrence was calculated from diagnosis to tumor recurrence.

\section{Statistical analysis}

All statistical analyses were performed using IBM SPSS Version 23.0 software (IBM Corporation, Armonk, New York, USA). Descriptive statistics were used to characterize the study cohort. The cut off points of factors were set by the median number for both continuous variables and categorical variables. The prognostic factors for recurrence time was compared to establish subgroups of the factors. Kaplan-Meier analysis was used to study survival, and the factors with statistical significance were included in the Cox regression analysis. Hazard ratios (HR) with $95 \%$ confidence intervals $(\mathrm{CI})$ were reported. A P value $<0.05$ was considered statistically significant.

\section{Results}

A total of 176 adult patients with recurrent GBM diagnosed between 2008 and 2020 were identified. The patient characteristics are summarized in Table 1 . The median recurrence time was 10.0 months (range, 1-75 months). The median age at diagnosis was 57 years (range: 19-76 years), and 
Table 1 A summary of patient demographics, and clinical and radiological features

\begin{tabular}{lc}
\hline Characteristics & $\mathrm{N}(\%)$ \\
\hline Age $<57$ years & $88(50.0)$ \\
Gender (male) & $113(64.2)$ \\
Pre-KPS ( $\geq 90)$ & $101(57.4)$ \\
Location of tumor & \\
Frontal lobe & $96(54.5)$ \\
Temporal lobe & $47(26.7)$ \\
Parietal lobe & $21(11.9)$ \\
Occipital lobe & $11(6.3)$ \\
Tumor volume $\left(>33 \mathrm{~cm}^{3}\right)$ & $20(45.5)$ \\
Tumor diameter $\left(\geq 4.7 \mathrm{~cm}^{3}\right)$ & $23(52.3)$ \\
Contact with SVZ & $22(64.7)$ \\
Enhanced feature & \\
Ring & $5(16.7)$ \\
Heterogeneity & $30(83.6)$ \\
\hline
\end{tabular}

SVZ, subventricular zone.

males represented 113 (64.2\%) cases. The median KPS before treatment was 90 (range, 40-100). Tumors were detected in the frontal lobe $(n=96,54.5 \%)$, the temporal lobe $(n=47$, $26.7 \%)$, the parietal lobe ( $\mathrm{n}=21,11.9 \%)$, and the occipital lobe $(\mathrm{n}=11,6.3 \%)$. The median tumor diameter and volume were $4.5 \mathrm{~cm}$ and $33.0 \mathrm{~cm}^{3}$, respectively. Heterogeneous enhancement and ring-enhancement were observed in 30 patients and 5 patients, respectively. Twenty-two patients presented with GBM that had contact with the SVZ.

Immunohistochemistry showed positive staining for IDH1 in $6.9 \%$ of cases (4/58), GFAP in $95.8 \%(46 / 48)$, TP53 in $78.6 \%$ (33/42), Ki67 $\geq 0.3$ in $82.0 \%$ (41/50), TERT $\mathrm{C} 228 \mathrm{~T}$ in $33.3 \%(7 / 21)$, MGMT promoter methylation in $60.6 \%(20 / 33)$, ATRX in $16.0 \%$ (4/25), and BRAF V600E in $90.5 \%(19 / 21)$ of patients.

Surgical and adjuvant therapies for all patients are summarized in Table 2. Surgery consisted of GTR in 18 patients $(10.2 \%)$, STR in 53 patients $(30.1 \%)$, and PR in 105 patients $(59.7 \%)$. Postoperatively, all patients received RT and the median time from diagnosis to RT after surgery was 28.0 days (range, 1-333 days). A total of 97 patients received concurrent $\mathrm{CTh}$, adjuvant $\mathrm{CTh}$ was administered to 105 patients (59.7\%), and 69 patients received RT with concurrent and adjuvant CTh.
Table 2 A summary of the surgical and adjuvant therapies administered to the patients

\begin{tabular}{lc}
\hline Variable & $\mathrm{N}(\%)$ \\
\hline Extent of resection & $18(10.2)$ \\
GTR & $53(30.1)$ \\
STR & $105(59.7)$ \\
PR & \\
Adjuvant therapies & $176(100.0)$ \\
RT & $97(55.1)$ \\
RT + concurrent CTh & $105(59.7)$ \\
RT + adjuvant CTh & $69(39.2)$ \\
RT + concurrent and adjuvant CTh & $64(37.0)$ \\
Duration of CTh (4-6 weeks) & $82(50.0)$ \\
Radiation interval ( $\geq 28$ days) &
\end{tabular}

Univariate analysis of the whole cohort (Tables 3,4) demonstrated that age, gender, KPS, location, enhancement features, tumor diameter, tumor volume, radiation interval time, concurrent $\mathrm{CTh}$, adjuvant $\mathrm{CTh}$, and duration of $\mathrm{CTh}$ were not correlated with recurrence time $(\mathrm{P}>0.05)$. However, $\mathrm{PR}(\mathrm{P}=0.004)$, contact with the SVZ ( $\mathrm{P}=0.004)$, IDH1 wildtype $(\mathrm{P}=0.048)$, TERT C228T wild-type $(\mathrm{P}=0.012)$, and positive GFAP expression $(\mathrm{P}=0.044)$ were all associated with a shorter recurrence time. Multivariate analysis demonstrated that PR (HR 1.440, 95\% CI: 1.025-2.024, P=0.036), contact with the SVZ (HR 3.523, 95\% CI: 1.380-8.990, $\mathrm{P}=0.008$ ), and TERT C228T wild-type (HR 0.296, 95\% CI: 0.104$0.896, \mathrm{P}=0.023$ ) were independent unfavorable risk factors for short-term tumor recurrence (Table 5 ).

\section{Discussion}

GBM is a type of intracranial malignant tumor, among which supratentorial GBM is the most common. Standard therapy includes tumor resection, RT, and CTh. Previous studies have suggested that age, extent of resection, CTh, Ki67 proliferative index, and IDH mutation were correlated with overall survival (5-10). However, few studies have examined the factors that influencing the short-term recurrence of GBM. In this current report, the data of 176 GBM patients with tumor recurrence were analyzed to determine the risk factors related to short-term recurrence 
Table 3 Univariate analysis of the recurrence time in 176 recurrent glioblastoma patients

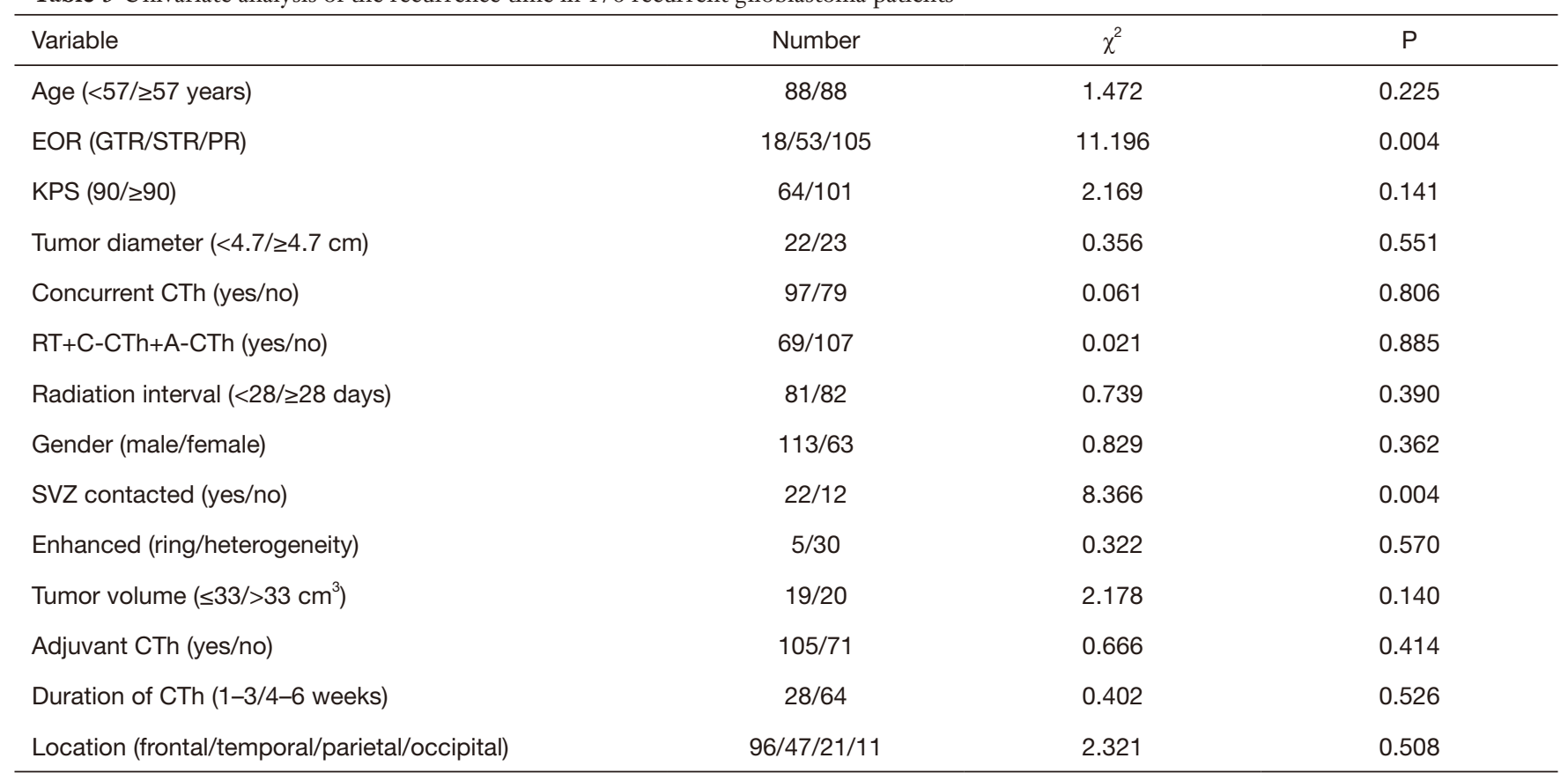

EOR, extent of resection; GTR, gross total resection; STR, subtotal resection; PR, partial resection; KPS, Karnofsky performance status; $\mathrm{RT}$, radiotherapy; CTh, chemotherapy; C-CTh, concurrent chemotherapy; A-CTh, adjuvant chemotherapy.

Table 4 Univariate analysis of the recurrence time in glioblastoma patients

\begin{tabular}{lccc}
\hline Variable & Number & $\chi^{2}$ & P \\
\hline IDH1 (wild-type/mut) & $54 / 4$ & 3.904 & 0.048 \\
TERT C228T (wild-type/mut) & $14 / 7$ & 6.360 & 0.012 \\
GFAP (positive/negative) & $46 / 2$ & 4.048 & 0.044 \\
Ki67 (<0.3/ $\geq 0.3)$ & $9 / 41$ & 1.252 & 0.263 \\
TP53 (wild-type/mut) & $8 / 33$ & 0.013 & 0.910 \\
ATRX (wild-type/mut) & $21 / 4$ & 0.003 & 0.959 \\
BRAF V600E (wild-type/mut) & $2 / 19$ & 3.141 & 0.076 \\
MGMT promoter (no/methylation) & $20 / 13$ & 0.012 & 0.913 \\
\hline
\end{tabular}

IDH1, isocitrate dehydrogenase 1; GFAP, glial fibrillary acidic protein; TP53, tumor protein P53; BRAF, B-raf gene.

of GBM. Identifying these risk factors may provide novel insights into the recurrence of GBM.

\section{Age and gender}

In this study cohort, there were more males $(64.2 \%)$ than females $(35.8 \%)$. However, in agreement with previous reports $(9,10)$, there were no significant differences in recurrence time between the genders. The median age at diagnosis was 57.0 years, and no differences were detected between patients $\leq 57$ years and patients $>57$ years. This was inconsistent with previous studies (11) which suggested that younger patients have a better prognosis compared to elderly patients, and that age is an important factor affecting glioma recurrence $(10,12-14)$. This may be due to the specific population in our cohort where all patients were diagnosed with GBM.

\section{Neuroimaging characteristics}


Table 5 Cox regression analysis of the recurrence time in patients with recurrent glioblastoma

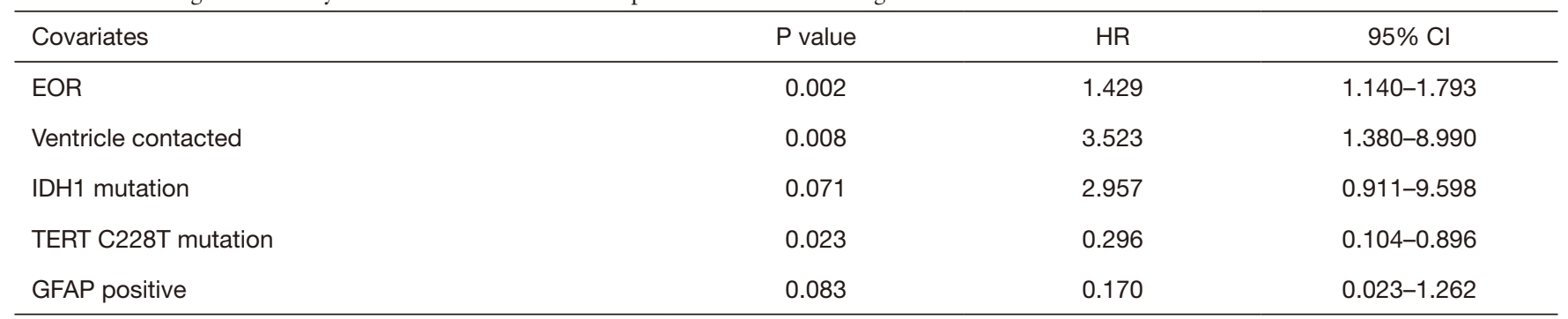

EOR, extent of resection; IDH1, isocitrate dehydrogenase 1, TERT, telomerase reverse transcriptase; GFAP, glial fibrillary acidic protein; $\mathrm{HR}$, hazard ratio; $\mathrm{Cl}$, confidence interval.

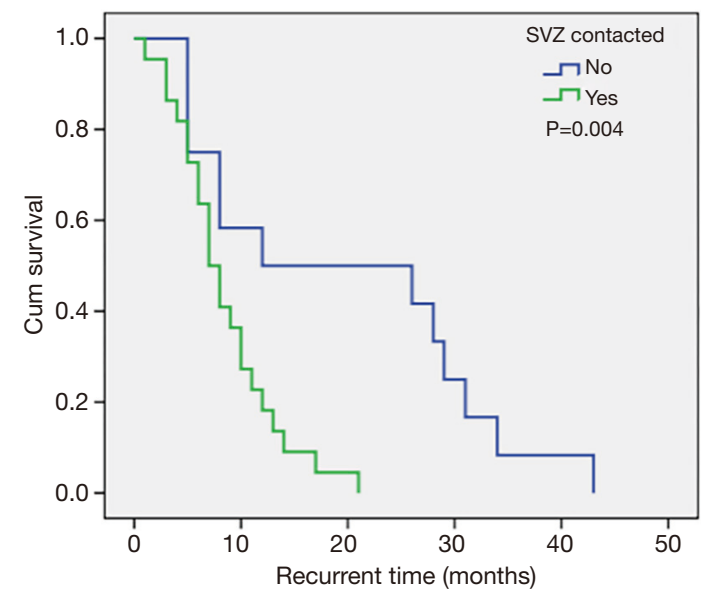

Figure 1 The tumor recurrence time was not significantly affected by whether the glioblastomas was in contact with the subventricular zone or not.

The most common sites of GBM were the frontal lobe, the temporal lobe, the parietal lobe, and the occipital lobe, successively from high to low. Approximately half of all GBMs were detected in the frontal lobe. However, there was no correlation between tumor location and recurrence time. Almost all GBMs had some degree of enhancement on contrast-enhanced MRI. In $67.4 \%$ of patients, imaging showed that the tumor had contact with the SVZ. These patients had a shortened recurrence time compared to patients without SVZ contact (median recurrence time: 12.0 vs. 7.0 months; Figure 1). Furthermore, SVZ contact was confirmed as an independent unfavorable risk factor to short-term tumor recurrence, and this was consistent with the observations by Comas et al. (15). Hallaert and colleagues (1) also analyzed the survival of 93 patients with GBM. In this latter study, $68 \%$ of patients had SVZ contact and the median progression free survival (PFS) was
5.9 months. This was significantly poorer compared to patient without SVZ contact. The SVZ contains multiple cell types with neural stem cells being the most abundant $(16,17)$, and these are considered a source of cancer cells that are related to tumor formation and gliomagenesis (18). Numerous studies have suggested that GBMs that have contact with the SVZ have more aggressive patterns of relapse and poorer outcomes (19-24).

\section{Molecular markers}

Molecular biomarkers are used widely in the WHO 2016 classification of central nervous system tumors (25), and several molecular biomarkers have been identified to have significant relationships with the prognosis of patients (26). In this study cohort, $31.8 \%$ of patients showed the TERT C228T mutation, and the median recurrence times were 5.0 months and 11.0 months in patients with the TERT C228T wild-type and mutation, respectively (Figure 2), suggesting that the TERT C228T wild-type was an independent risk factor for shortened tumor recurrence time. This is contrary to previous studies (8) and may be due to the limited sample size in our investigation and thus, this conclusion should be interpreted with caution.

The IDH1 mutation rate in our cohort was $6.9 \%$, and patients with the IDH1 mutation had a longer recurrence time compared to wild-type patients (median recurrence time: 18.0 vs. 8.0 months; Tables 3; Figure 3). However, the IDH1 mutation was not an independent risk factor for tumor recurrence. The IDH mutation is considered an independent predictor of good prognosis in all types of glioma patients, and patients with the IDH1 mutation generally have a prolonged PFS $(27,28)$. However, Gülten et al. (29) summarized the IDH expression characteristics of 83 GBM patients and found that the IDH mutation rate 


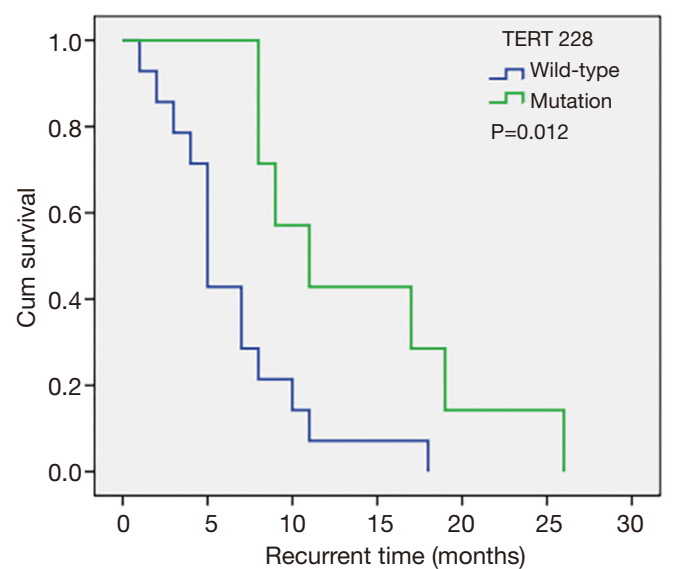

Figure 2 Patients with the telomerase reverse transcriptase (TERT) C228T mutation had a shortened recurrence time.

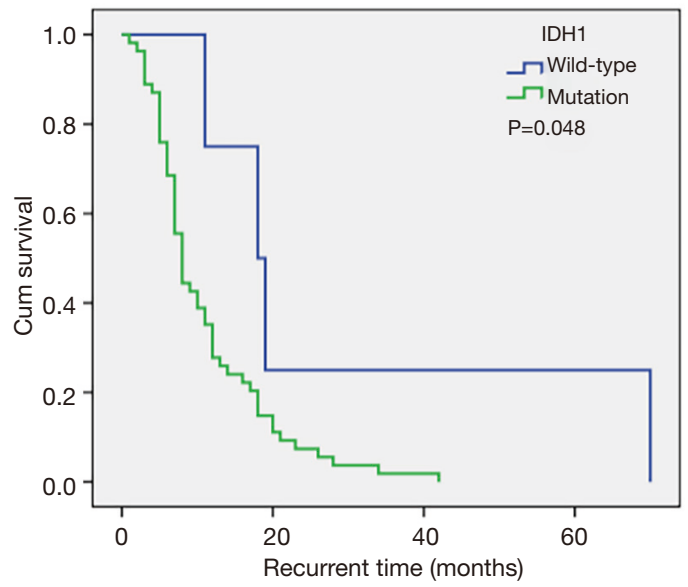

Figure 3 The isocitrate dehydrogenase 1 (IDH1) mutation was associated with a longer recurrence time.

was low and that it was not a factor affecting the recurrence time (29). In the study by Potharaju and colleagues (8), patients with the IDH mutation had a prolonged recurrence time, however, it was not an independent risk factor for tumor recurrence, and this is consistent with our results.

A total of $95.8 \%$ of our patients were positive for GFAP. The recurrence time in patients with negative GFAP was longer compared to patients who were positive for GFAP (Figure 4). However, GFAP was not an independent factor for tumor recurrence. In some studies $(9,30,31)$, the Ki-67 proliferative index has been shown to be an independent prognostic factor for time to recurrence. In contrast, in the current study, the Ki-67 proliferative index was not related

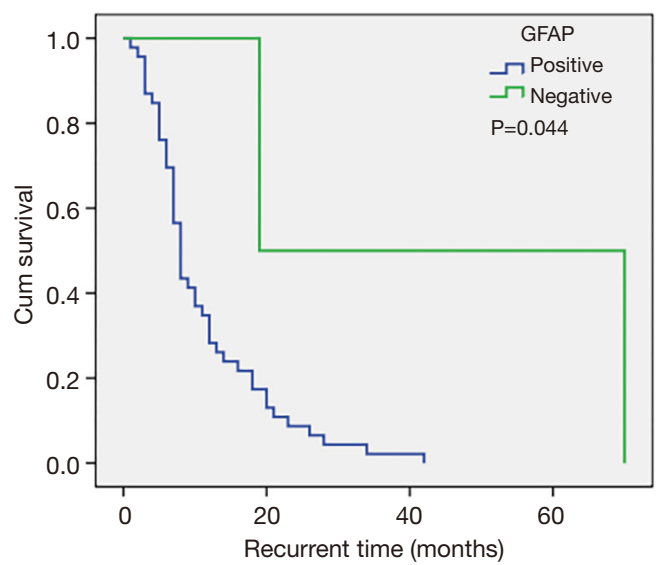

Figure 4 Patients with negative glial fibrillary acidic protein (GFAP) expression had a longer recurrence time.

to recurrence time, and this may be due to the entire cohort being GBM patients GBM patients generally have a high $\mathrm{Ki}-67$ proliferative index and in fact, $82 \%$ of the patient cohort showed a Ki-67 proliferative index $\geq 30 \%$.

\section{Extent of resection, $R T$, and $C T h$}

The maximal feasible safe resection is the guiding principle for GBM surgery $(2,32)$. The EOR partially depends on the size and location of the tumor. With advances in neuroimaging it is possible to assess the relationship between normal brain tissues and tumor tissues postoperatively. However, when the tumor invades important functional areas, it is difficult to perform GTR. In this current study, the median recurrence times for patients with GTR, STR, and PR were 14.0 months, 13.0 months, and 8.5 months, respectively (Figure 5). PR was confirmed as an independent risk factor for short-term tumor recurrence, which concurs with previously published studies. The extent of surgery is an independent factor for recurrence time and overall survival regardless of molecular status (2). Therefore, surgical resection is considered an important step in the management of GBM.

RT can improve both local control and outcome, and has long been used in the management of GBM (2). Indeed, RT was administered to all patients in this study and thus, the relationship between RT and recurrence time could not be determined. However, the median recurrence time (10.0 months) was longer than that reported in previous studies (33), suggesting that to some extent, our patients 


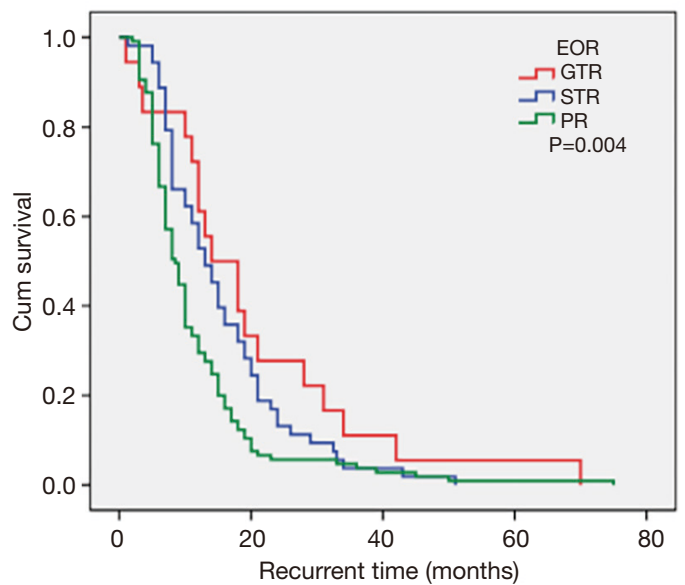

Figure 5 The extent of resection (EOR) had a statistically significant effect on recurrence time. GTR, gross total resection; STR, subtotal resection; PR, partial resection.

benefited from RT. Some reports have noted that delayed adjuvant chemoradiation ( $\geq 20$ days) was an unfavorable factor for survival outcome (7). However, no relationship between RT interval time was observed in our results. This may due to most of our patients receiving RT more than 20 days after surgery. The first-line standard CTh includes temozolomide (TMZ) during RT followed by a further six cycles of TMZ (32). In this current report, $55.1 \%$ of patients received concurrent CTh during RT, adjuvant $\mathrm{CTh}$ was administered to $59.7 \%$ of patients, and RT combined with concurrent and adjuvant $\mathrm{CTh}$ was performed in $39.2 \%$ of cases. As most of our patients did not received 6 cycles of TMZ, the duration of CTh was divided into two groups, namely, 1-3 weeks and 4-6 weeks. The factors of concurrent CTh and adjuvant CTh had no effect on the recurrence time. In the majority of reports (31), completing 6 cycles of adjuvant TMZ is a favorable factor for PFS. In our study, $96.7 \%$ of our patients had less than 6 cycles of adjuvant TMZ, and this may explain the lack of effect of $\mathrm{CTh}$ on recurrence time. Although patients who received 6 or more cycles of adjuvant TMZ had a longer recurrence time, drug toxicity was also increased, and this can lead to decreased quality of life, especially in elderly patients (34). Therefore, the role of adjuvant CTh in survival outcome remains controversial.

\section{Study strengths and limitations}

This report identified the prognostic factors affecting the recurrence time in patients with GMB. However, the study has some limitations. First, the sample size of the molecular features is limited and the results may not reflect the actual situation in a larger population. Second, all our patients received RT and it was not possible to analyze the relationship between RT and recurrence time. Third, this study identified the TERT C228T wild-type as an independent unfavorable factor for recurrence time which was not consistent with previous studies and this conclusion should be interpreted with caution. Fourth, further studies are recommended to investigate the relationship among the status of Ki-67, TERT, the expression patterns of biomarkers, and time to relapse after surgery.

\section{Conclusions}

Patients with PR, SVZ contact, IDH1 wild-type, TERT C228T mutation, and positive GFAP had a shorter recurrence time compared with the corresponding control groups. The key factors affecting the recurrence time in GMB patients were EOR, SVZ contact, and TERT C228T status. The state of pathological molecular markers has guiding significance for adjuvant therapy. In addition, comprehensive therapy should be given regardless of molecular status, especially the maximum degree of safe surgical resection of the tumor.

\section{Acknowledgments}

Funding: None.

\section{Footnote}

Reporting Checklist: The authors have completed the STROBE reporting checklist. Available at http://dx.doi. org/10.21037/apm-21-823

Data Sharing Statement: Available at http://dx.doi. org/10.21037/apm-21-823

Conflicts of Interest: All authors have completed the ICMJE uniform disclosure form (available at http://dx.doi. org/10.21037/apm-21-823). The authors have no conflicts of interest to declare.

Ethical Statement: The authors are accountable for all aspects of the work in ensuring that questions related to the accuracy or integrity of any part of the work are 
appropriately investigated and resolved. All procedures performed in this study involving human participants were in accordance with the Declaration of Helsinki (as revised in 2013). The study was approved by the Ethics Committee of Renji Hospital, Shanghai Jiaotong University School of Medicine (No. RA-2020-056). Individual consent for this retrospective analysis was waived.

Open Access Statement: This is an Open Access article distributed in accordance with the Creative Commons Attribution-NonCommercial-NoDerivs 4.0 International License (CC BY-NC-ND 4.0), which permits the noncommercial replication and distribution of the article with the strict proviso that no changes or edits are made and the original work is properly cited (including links to both the formal publication through the relevant DOI and the license). See: https://creativecommons.org/licenses/by-nc-nd/4.0/.

\section{References}

1. Hallaert G, Pinson H, Van den Broecke C, et al. Subventricular zone contacting glioblastoma: tumor size, molecular biological factors and patient survival. Acta Oncol 2020;59:1474-9.

2. Tan AC, Ashley DM, Lopez GY, et al. Management of glioblastoma: State of the art and future directions. CA Cancer J Clin 2020;70:299-312.

3. Delgado-Fernández J, Frade-Porto N, Blasco G, et al. Does reintervention improve survival in recurrent glioblastoma? Facing a temporal bias in the literature. Acta Neurochir 2020;162:1967-75.

4. Lacroix M, Abi-Said D, Fourney DR, et al. A multivariate analysis of 416 patients with glioblastoma multiforme: prognosis, extent of resection, and survival. J Neurosurg 2001;95:190-8.

5. De Bonis $\mathrm{P}$, Fiorentino A, Anile C, et al. The impact of repeated surgery and adjuvant therapy on survival for patients with recurrent glioblastoma. Clin Neurol Neurosurg 2013;115:883-6.

6. Tully PA, Gogos AJ, Love C, et al. Reoperation for Recurrent Glioblastoma and Its Association With Survival Benefit. Neurosurgery 2016;79:678-89.

7. Potharaju M, Mathavan A, Mangaleswaran B, et al. Delay in adjuvant chemoradiation impacts survival outcome in glioblastoma multiforme patients. Acta Oncol 2020;59:320-3.

8. Potharaju M, Mathavan A, Mangaleswaran B, et al. Clinicopathological Analysis of HIF-1alpha and TERT on
Survival Outcome in Glioblastoma Patients: A Prospective, Single Institution Study. J Cancer 2019;10:2397-406.

9. Li J, Niu X, Gan Y, et al. Clinical and Pathologic Features and Prognostic Factors for Recurrent Gliomas. World Neurosurg 2019;128:e21-30.

10. Yang P, Wang Y, Peng X, et al. Management and survival rates in patients with glioma in China (2004-2010): a retrospective study from a single-institution. J Neurooncol 2013;113:259-66.

11. Reavey-Cantwell JF, Haroun RI, Zahurak M, et al. The prognostic value of tumor markers in patients with glioblastoma multiforme: analysis of 32 patients and review of the literature. J Neurooncol 2001;55:195-204.

12. Smith JS, Chang EF, Lamborn KR, et al. Role of extent of resection in the long-term outcome of low-grade hemispheric gliomas. J Clin Oncol 2008;26:1338-45.

13. Nuño M, Birch K, Mukherjee D, et al. Survival and prognostic factors of anaplastic gliomas. Neurosurgery 2013;73:458-65; quiz 465.

14. Lee KJ, Marchan E, Peterson J, et al. Management and Survival of Adult Patients with Pilocytic Astrocytoma in the National Cancer Database. World Neurosurg 2018;112:e881-7.

15. Comas S, Luguera E, Molero J, et al. Influence of glioblastoma contact with the subventricular zone on survival and recurrence patterns. Clin Transl Oncol 2021;23:554-64.

16. Doetsch F, Garcia-Verdugo JM, Alvarez-Buylla A. Cellular composition and three-dimensional organization of the subventricular germinal zone in the adult mammalian brain. J Neurosci 1997;17:5046-61.

17. Dulken BW, Leeman DS, Boutet SC, et al. Single-Cell Transcriptomic Analysis Defines Heterogeneity and Transcriptional Dynamics in the Adult Neural Stem Cell Lineage. Cell Rep 2017;18:777-90.

18. Tarizzo ML, Nataf R. The treatment of trachoma. Rev Int Trach 1969-1970;46:7-99.

19. Chaichana KL, McGirt MJ, Frazier J, et al. Relationship of glioblastoma multiforme to the lateral ventricles predicts survival following tumor resection. J Neurooncol 2008;89:219-24.

20. Jafri NF, Clarke JL, Weinberg V, et al. Relationship of glioblastoma multiforme to the subventricular zone is associated with survival. Neuro Oncol 2013;15:91-6.

21. Young GS, Macklin EA, Setayesh K, et al. Longitudinal MRI evidence for decreased survival among periventricular glioblastoma. J Neurooncol 2011;104:261-9.

22. Adeberg S, Konig L, Bostel T, et al. Glioblastoma 
recurrence patterns after radiation therapy with regard to the subventricular zone. Int J Radiat Oncol Biol Phys 2014;90:886-93.

23. Chen L, Chaichana KL, Kleinberg L, et al. Glioblastoma recurrence patterns near neural stem cell regions. Radiother Oncol 2015;116:294-300.

24. Lim DA, Cha S, Mayo MC, et al. Relationship of glioblastoma multiforme to neural stem cell regions predicts invasive and multifocal tumor phenotype. Neuro Oncol 2007;9:424-9.

25. Louis DN, Perry A, Reifenberger G, et al. The 2016 World Health Organization Classification of Tumors of the Central Nervous System: a summary. Acta Neuropathol 2016;131:803-20.

26. D'Amico RS, Englander ZK, Canoll P, et al. Extent of Resection in Glioma-A Review of the Cutting Edge. World Neurosurg 2017;103:538-49.

27. Eckel-Passow JE, Lachance DH, Molinaro AM, et al. Glioma Groups Based on 1p/19q, IDH, and TERT Promoter Mutations in Tumors. N Engl J Med 2015;372:2499-508.

28. Noushmehr H, Weisenberger DJ, Diefes K, et al. Identification of a $\mathrm{CpG}$ island methylator phenotype that defines a distinct subgroup of glioma. Cancer Cell 2010;17:510-22.

Cite this article as: Huang R, Wang T, Liao Z, Wang Z, Ye M, Zhou D, Xie H, Bai Y, Qiu Y, Liu Y. A retrospective analysis of the risk factors affecting recurrence time in patients with recurrent glioblastoma. Ann Palliat Med 2021;10(5):5391-5399. doi: 10.21037/apm-21-823
29. Gülten G, Yalcin N, Baltalarli B, et al. The importance of IDH1, ATRX and WT-1 mutations in glioblastoma. Pol J Pathol 2020;71:127-37.

30. Freije WA, Castro-Vargas FE, Fang Z, et al. Gene expression profiling of gliomas strongly predicts survival. Cancer Res 2004;64:6503-10.

31. Alimohammadi E, Bagheri SR, Sadeghsalehi A, et al. Prognostic factors in patients with glioblastoma multiforme: focus on the pathologic variants. Acta Neurol Belg 2020;120:1341-1350. Erratum in: Acta Neurol Belg 2020;120:1497.

32. Stupp R, Mason WP, van den Bent MJ, et al. Radiotherapy plus concomitant and adjuvant temozolomide for glioblastoma. N Engl J Med 2005;352:987-96.

33. Youssef M, Ludmir EB, Mandel JJ, et al. Treatment strategies for glioblastoma in older patients: age is just a number. J Neurooncol 2019;145:357-64.

34. Balana C, Vaz MA, Sepulveda JM, et al. A phase II randomized, multicenter, open-label trial of continuing adjuvant temozolomide beyond six cycles in patients with glioblastoma (GEINO 14-01). Neuro Oncol 2020;22:1851-61.

(English Language Editor: B. Draper) 